\title{
Identification of Featured Metabolism-Related Genes in Patients with Acute Myocardial Infarction
}

\author{
Hang Xie, Enfa Zha $\mathbb{D}$, and Yushun Zhang $\mathbb{D}$ \\ Department of Structural Heart Disease, The First Affiliated Hospital of Xi'an Jiaotong University, Xi'an 710061, China
}

Correspondence should be addressed to Yushun Zhang; zys2889@sina.com

Received 11 September 2020; Revised 5 November 2020; Accepted 11 November 2020; Published 28 November 2020

Academic Editor: Agata Sakowicz

Copyright @ 2020 Hang Xie et al. This is an open access article distributed under the Creative Commons Attribution License, which permits unrestricted use, distribution, and reproduction in any medium, provided the original work is properly cited.

\begin{abstract}
Objective. A growing body of emerging evidence indicates that metabolic processes play a pivotal role in the biological processes underlying acute myocardial infarction (AMI). The aim of the current study was to identify featured metabolism-related genes in patients with AMI using a support vector machine (SVM) and to further explore the value of these genes in the diagnosis of AMI. Methods. Gene microarray expression data related to AMI were downloaded from the GSE66360 dataset in the Gene Expression Omnibus (GEO) database. This data set consisted of 50 AMI samples and 49 normal controls that were randomly classified into a discovery cohort (21 AMI samples and 22 normal controls) and a validation cohort (28 AMI and 28 normal controls). We applied a machine learning method that combined SVM with recursive feature elimination (RFE) to discriminate AMI patients from normal controls. Based on this, an SVM classifier was constructed. Receiver operating characteristic (ROC) analysis was used to investigate the predictive value for the early diagnosis of AMI in the two cohorts and was then further verified in an independent external cohort. Results. Three metabolism-related genes were identified based on SVM-RFE (AKR1C3, GLUL, and PDE4B). The SVM classifier based on the three genes allowed for excellent discrimination between AMI and healthy samples in both the discovery cohort $(\mathrm{AUC}=0.989)$ and the validation cohort $(\mathrm{AUC}=0.964)$, and this was further confirmed in the GSE68060 dataset (AUC = 0.839). Additionally, the SVM classifier allowed for perfect discrimination between recurrent AMI events and nonrecurrent events in the GSE68060 cohort (AUC = 0.992). GO and KEGG pathway enrichment analysis of the identified featured genes revealed significant enrichment of specific metabolic pathways. Conclusion. The identified metabolism-related genes may play important roles in the development of AMI and may represent diagnostic and therapeutic biomarkers of AMI.
\end{abstract}

\section{Introduction}

AMI results from interrupted blood flow to a certain area of the heart and is considered one of the primary causes of disability and death from cardiovascular disease worldwide, thus posing a serious threat to human health [1]. Over the last decade, the primary therapeutic strategies, including percutaneous coronary intervention, coronary artery bypass surgery, and medications, have improved the prognosis of AMI. However, approximately one-third of eligible patients failed to receive early reperfusion therapy due to late detection [2]. Early diagnosis and interventional therapy are beneficial in that they significantly reduce mortality and improve prognosis [3]. Therefore, an early diagnosis may markedly contribute to overall survival.
The diagnosis of AMI is typically based upon the observation of changes in a surface electrocardiogram (ECG) and blood levels of sensitive and specific biomarkers such as cTnI/T and CKMB. However, the sensitivity and specificity of these biomarkers remain unsatisfactory, often resulting in a lack of diagnosis or misdiagnosis $[3,4]$. Based on this, potential biomarkers possessing high sensitivity and specificity for early diagnosis of AMI are urgently required and could ultimately contribute to improved clinical survival. Gene expression profiles related to AMI have been previously studied. The differentially expressed genes related to cardiovascular events exhibit similar variation components to those of AMI-related genes. Regardless of if they are upregulated or downregulated, they change in the same direction [5]. This study suggests that differentially expressed genes may provide a new biomarker 
for predicting AMI. Recently, metabolic pathways in cardiovascular disease have been demonstrated to represent potential new valuable targets for drug therapy $[6,7]$. A recent study revealed that exercise improves cardiac function and glucose metabolism in mice with experimental myocardial infarction by inhibiting phosphorylated histone deacetylase 4 (HDAC4) and upregulating glucose transporter 1 (GLUT1) expression. These results demonstrated that metabolic processes play a pivotal role in the biological processes underlying AMI. Metabolism-related genes have been studied in the context of cardiac ischemia. By inhibiting miR-92a-3p, LNA-92a can increase endothelial cell autophagy and regulate the expression of metabolism-related genes, thereby increasing myocardial fatty acid uptake and mitochondrial function. These prosurvival mechanisms may reduce tissue damage after myocardial infarction [8]. However, the role of metabolismrelated genes in AMI remains to be fully elucidated.

In this study, differentially expressed metabolism-related genes were identified in normal and AMI samples. Next, an SVM classifier consisting of three risk genes was established. This classifier allowed patient samples to be distinguished from normal controls.

\section{Materials and Methods}

2.1. Microarray Data. To investigate metabolism-related genes, the microarray data for AMI were collected from the GEO (http://www.ncbi.nlm.nih.gov/geo/) database under the accession number GSE66360, where 50 AMI samples and 49 normal controls were included. The dataset was divided into a discovery cohort (21 AMI samples and 22 normal controls) and a validation cohort (28 AMI samples and 28 normal controls). Furthermore, the GSE48060 dataset consisting of 31 AMI patients and 21 healthy controls was obtained to confirm the performance of the SVM classifier. Additionally, 5 recurrence and 26 no-recurrence samples over a 1.5-year follow-up period were included in the GSE48060 dataset. All datasets were produced using the Affymetrix Human Genome U133 Plus 2.0 Array. Background correction and normalization were performed using linear models for the microarray data (LIMMA) software package. Normalization between arrays was performed using the quantile algorithm in LIMMA.

2.2. Metabolism-Related Genes. Metabolism-related genes were obtained from the Molecular Signatures Database v7.1 (MSigDB) (http://software.broadinstitute.org/gsea/msigdb) by searching using the term "metabolism." The C2 (c2.cp.kegg.v7.1.symbols.gmt) subcollection was selected as the reference gene set (Supplementary Table 1). A total of 948 unique metabolism-related genes were obtained. Next, we extracted the metabolism-related gene expression matrix from the GSE66360 dataset using the $\mathrm{R}$ language merge package. Finally, a gene expression matrix consisting of 862 gene expression values was obtained in the discovery cohort.

\subsection{Screening of Differentially Expressed Metabolism-Related} Genes. The analysis of differentially expressed metabolismrelated genes between AMI and normal samples was con- ducted using the LIMMA package implemented in the $\mathrm{R}$ statistical package (http://www.r-project.org). The threshold for the identification of differentially expressed genes was set at a $P$ value of $<0.05$ and a $\mid \log 2$ fold change (FC) $\mid \geq 1$.

2.4. Featured Gene Selection and the SVM Classifier Construction. The featured selection technique is an efficient tool for identifying meaningful information from a given gene dataset [9]. SVM is a supervised learning model that is aimed at classifying data points by maximizing the distance of a hyperplane for classification and regression analysis with high accuracy [10]. SVM-RFE is a popular feature selection technique and has exhibited promising and expanding applications for the analysis of high-dimensional data. It is much more robust with regard to data overfitting and classification accuracy than many other feature selection methods, and this technique has demonstrated its power in many fields, including metabolomics [11-13]. Therefore, we applied a machine learning method that combined SVM with RFE to select the best parameters for gene selection among all differentially expressed metabolism-related genes. Using this algorithm, optional feature genes were identified as risk genes in the context of AMI. Next, the identified feature genes were added into an SVM classifier with a radial basis function (RBF) kernel and 5-fold cross-validation to achieve predictions. To test the value of the identified featured genes, a heat map was clustered using the pheatmap package in $\mathrm{R}$ for all samples in the two cohorts (clustering method = "ward"). The Euclidean distance was used to cluster samples. Furthermore, the discriminatory power of the SVM classifier was measured according to the AUC (defined as the area under the receiver operating curve) in both cohorts, and this was further validated in the independent external cohort. Additionally, the performance of the SVM classifier was further explored in terms of AMI recurrence and nonrecurrence.

\subsection{Functional Enrichment Analysis of Identified Feature} Genes. To explore the functions and pathways of the identified feature genes, gene ontology (GO) and Kyoto Encyclopedia of Genes and Genomes (KEGG) pathway enrichment analyses were performed to identify potential functional components and pathways underlying numerous genes using the clusterProfiler package [14]. $P<0.05$ was considered to be statistically significant.

2.6. Statistical Analysis. The differentially expressed metabolism-related genes were identified using the Limma package with $P<0.05$ and $\mid \log 2$ fold change (FC) $\mid \geq 1$ as the cut-off criteria. Featured gene selection was performed using the RFE function in the caret package with 5-fold cross-validation. The SVM classifier was constructed using R package e1071 with 5-fold cross-validation. Hierarchical clustering analysis was used for the identified featured genes using the pheatmap package in R. ROC analysis was performed, and the area under the curve (AUC) was calculated to evaluate the predictive performance of the classifier. $P<0.05$ was considered to indicate a statistically significant difference. All statistical analyses were performed using $\mathrm{R}$ software (version 3.6.3, http://www.r-project.org). 


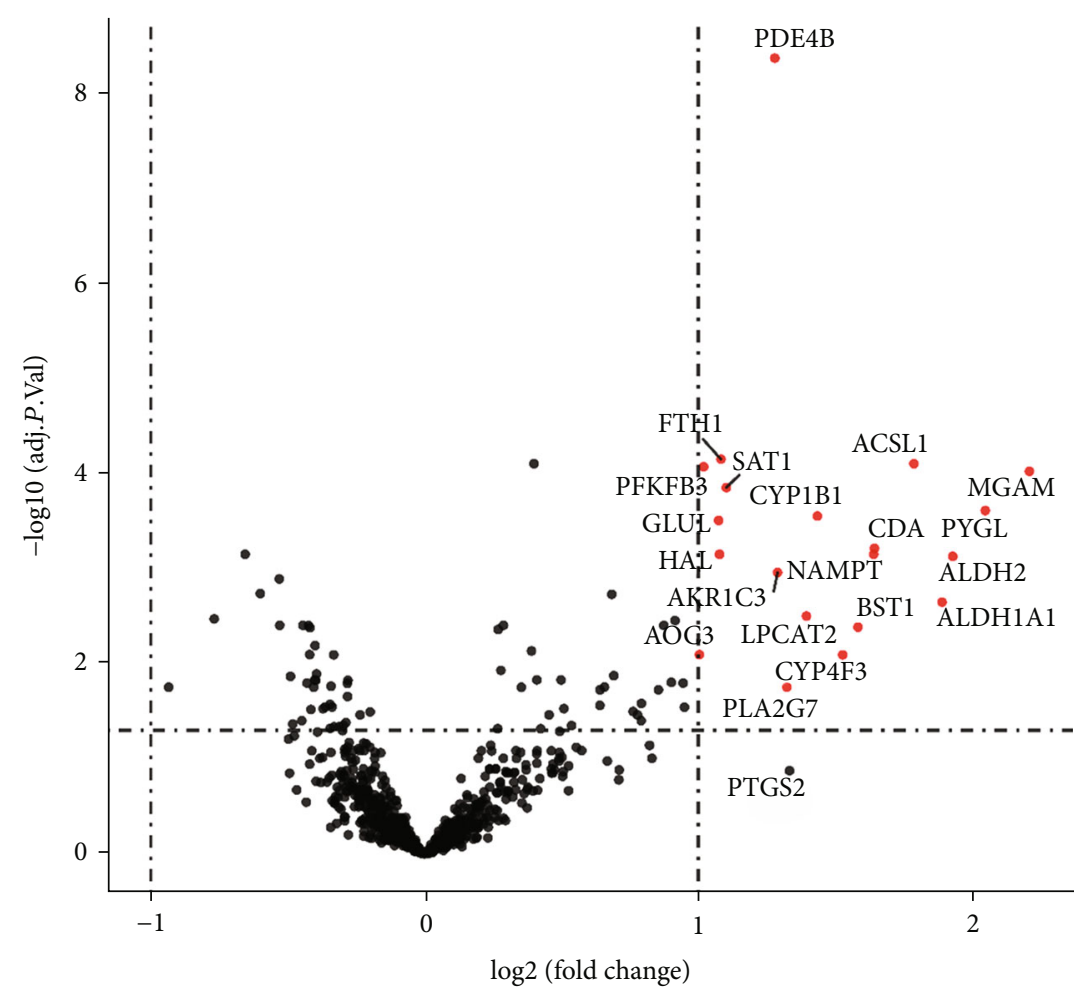

Significant
- False
- True

FIGURE 1: Differential expression of metabolic-related genes in AMI tissue and normal samples.

\section{Results}

3.1. Identification of Differentially Expressed MetabolismRelated Genes and Feature Genes. A total of 17 differentially expressed upregulated genes were identified between AMI tissues and normal tissues (Figure 1.). Based on the SVM-RFE algorithm, three genes (AKR1C3, GLUL, and PDE4B) with minimum root mean square error were fit into the SVM classifier (Figure 2). Hierarchical clustering analysis in the discovery cohort (Figure 3(a)) and the validation cohort (Figure 3(b)) revealed that patients could be clearly separated into two clusters based on the expression levels of the three identified feature genes. To validate the expression levels of three featured genes, the identified featured genes were confirmed in the validation cohort. As shown in Figure 4, the expression levels of two featured genes (GLUL and $P D E 4 B)$ in AMI tissues were significantly higher than those in the control group $(P<0.05)$.

3.2. Diagnostic Value of Three Feature Genes in AMI. As presented in Figure 5(a), the results of the 5 -fold cross-validation illustrated that the SVM classifier allowed for good classification in the discovery cohort between AMI and normal controls with an AUC of 0.989 (95\% CI = 0.966-1.00), a sensitivity of $95.24 \%$, and a specificity of $100.00 \%$. The SVM classifier demonstrated excellent discriminatory ability in the validation cohort with an AUC of 0.964 (95\% CI $=0.925$

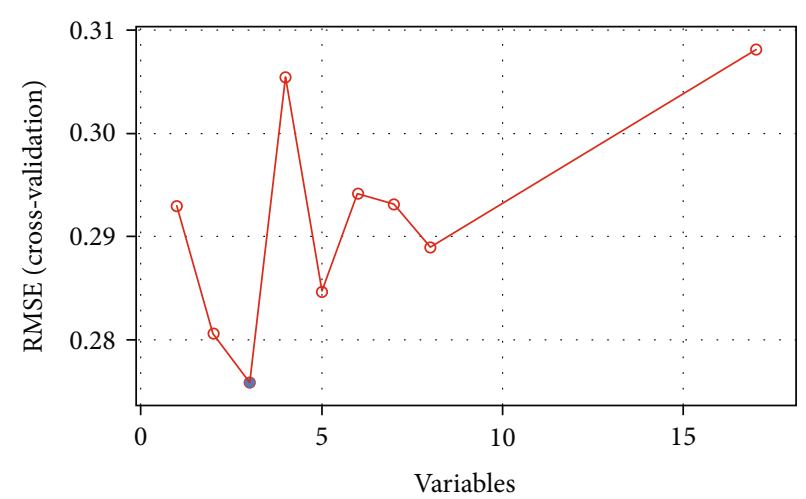

FIgURE 2: A plot of feature metabolic-related gene selection by recursive feature elimination.

$-1.000)$, a sensitivity of $85.71 \%$, and a specificity of $92.86 \%$ (Figure 5(b)). The discrimination power was confirmed in the independent GSE48060 cohort with an AUC of 0.839 (95\% CI $=0.715-0.962)$, a sensitivity of $83.87 \%$, and a specificity of $90.95 \%$ (Figure 5(c)). Furthermore, we investigated the discrimination ability of the classifier in the context of recurrent AMI. The classifier exhibited outstanding discrimination ability of recurrent AMI with an AUC of 0.992 (95\% $\mathrm{CI}=0.971-1.00)$, a sensitivity of $100 \%$, and a specificity of 96.15\% (Figure 5(d)). 


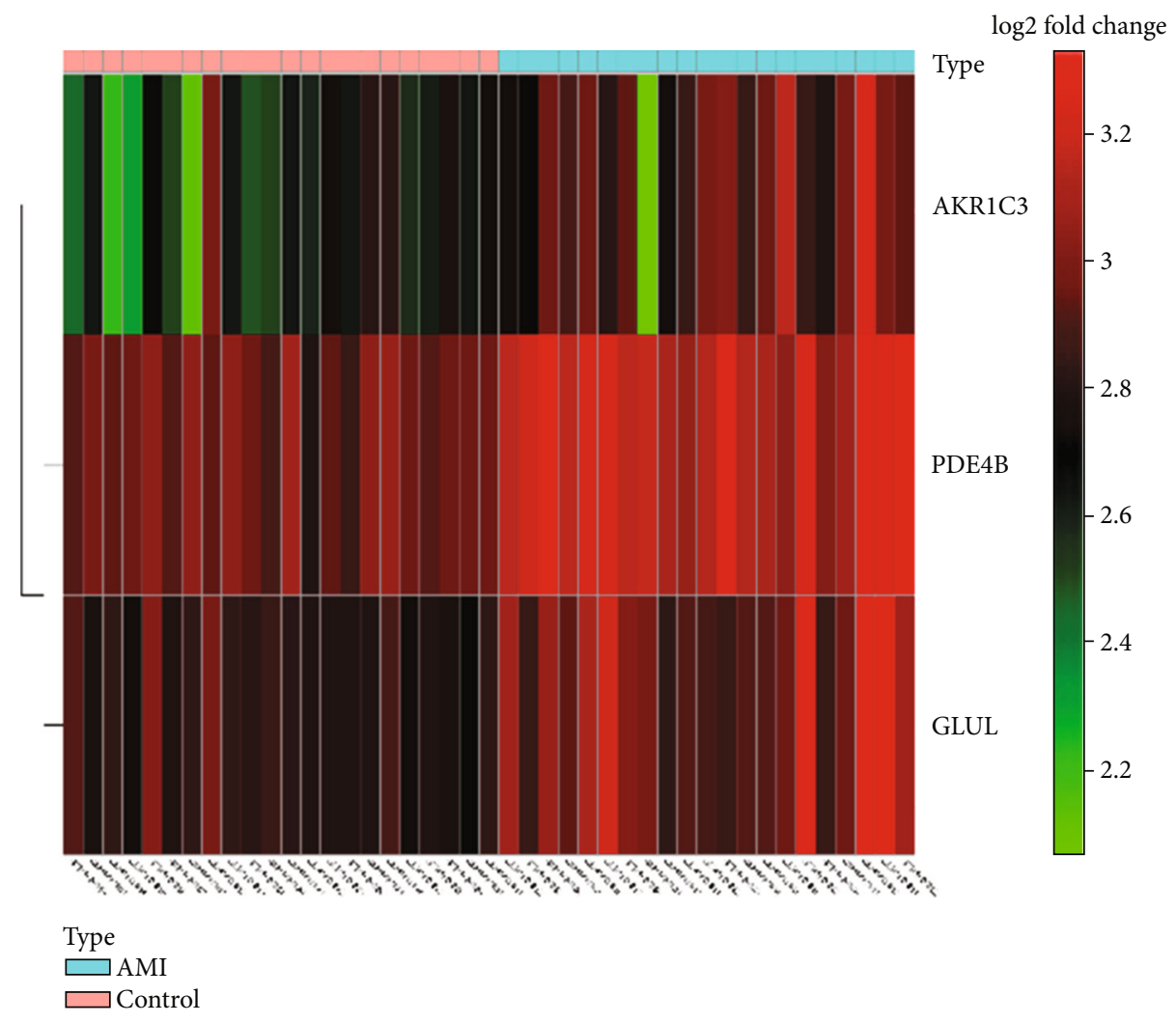

(a)

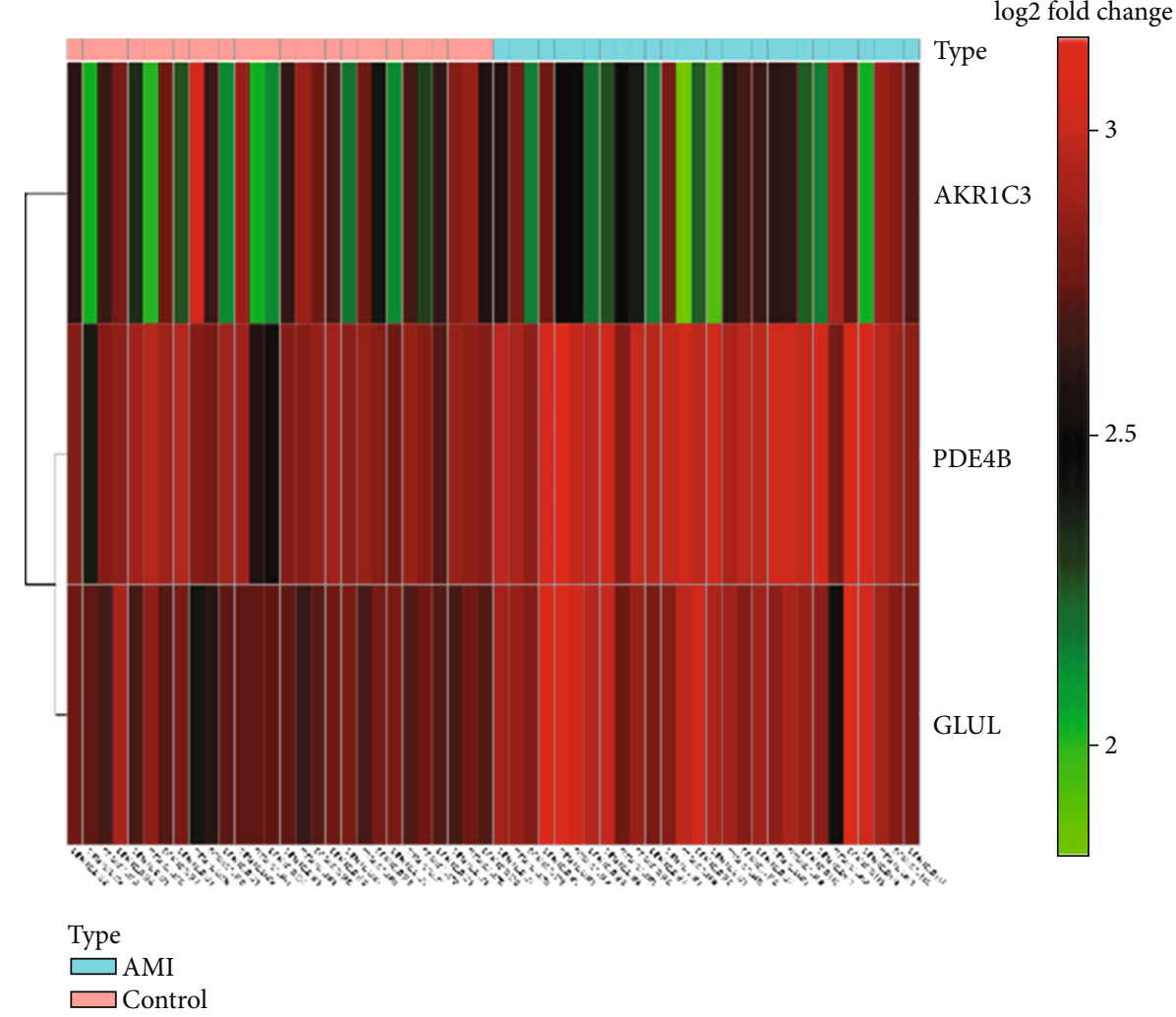

(b)

FIGURE 3: Hierarchical clustering analysis demonstrates identified metabolic-related gene expression patterns between AMI and normal tissues in the discovery cohort (a) and validation cohort (b). 


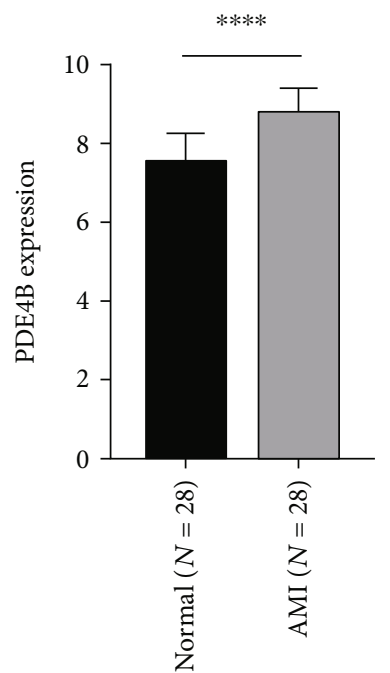

(a)

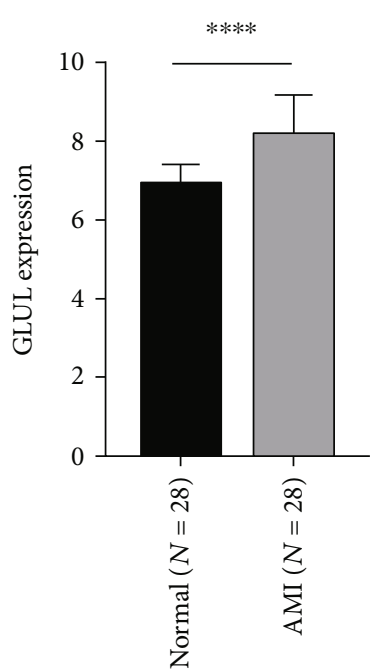

(b)

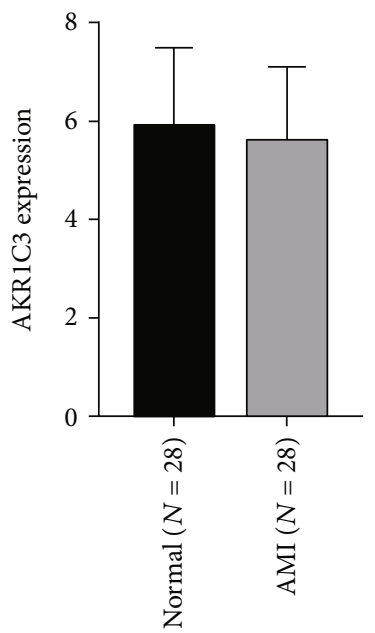

(c)

FIGURE 4: Validation of expression level of identified feature genes in patients with AMI and normal tissues in the validation cohort. (a) PDE4B, (b) GLUL, and (c) AKR1C3.

3.3. Functional Analysis of Feature Genes. Based on our results, cellular response to starvation, cellular response to nutrient levels, cellular response to extracellular stimulus, diterpenoid biosynthetic process, small molecule catabolic process, cyclooxygenase pathway, and negative regulation of hormone metabolic processes were the most significantly enriched biological processes (Figure 6(a)). Additionally, nitrogen metabolism, arginine biosynthesis, and folate biosynthesis were considered to be the most remarkably enriched pathways (Figure 6(b)).

\section{Discussion}

AMI remains a primary cause of death and disability worldwide despite significant improvements in diagnosis. The inhospital mortality for AMI remains high [15]. Recently, metabolism-related processes have been reported to be involved in the pathogenesis of AMI $[7,16]$. PRODH overexpression increases the number of gene transcripts related to metabolism, and this gene is related to the maintenance of normal mitochondrial function, ATP level, and redox homeostasis of human cardiomyocytes in a hypoxic environment [17]. However, the potential role of metabolism-related genes in AMI remains poorly understood. Single-nucleotide polymorphisms (SNPs) in some lipid metabolism-related genes are closely related to blood lipids and can cause coronary artery disease [18]. A study from Pakistan revealed that SNPs in lipid metabolism genes are significantly associated with MI susceptibility [19]. Circadian rhythm disorders can cause worsening of atherosclerosis [20]. A large number of metabolism-related genes exhibit a circadian rhythm [21]. Zhu et al. found that abnormal light can aggravate the circadian rhythm of lipid metabolism genes [22]. The above studies indicate that metabolic genes may increase the risk of AMI by affecting lipid metabolism.

To identify the metabolism-related genes that are involved in AMI, GSE66360 datasets were used to screen differentially expressed genes in patient tissues and control tissues. By comparing the expression levels of metabolismrelated genes between AMI patients and healthy samples, we found that 17 genes were differentially expressed in AMI compared to healthy samples, indicating that metabolism-related genes may play critical roles in the occurrence of AMI. Next, three featured genes in AMI samples were identified using the SVM-RFE algorithm that allows AMI samples to be distinguished from normal samples. The SVM classifier based on the identified featured genes allowed for good classification with an AUC of 0.989 for the patient samples. The discrimination power values of the classifiers for the validation cohort and the independent validation cohort were 0.964 and 0.839 , respectively. Furthermore, the SVM classifier can successfully distinguish patients with recurrent and nonrecurrent AMI with an AUC of 0.992. Therefore, the present study suggested that the featured genes could provide useful markers for identifying patients with AMI.

The present study demonstrated the potential value of metabolic-related genes in the context of AMI in the clinical setting. GLUL, PDE4B, and AKR1C3 were identified as potential metabolism-related genes that were associated with AMI and the recurrence of AMI. Glutamate-ammonia ligase (GLUL) belongs to the glutamine synthetase family and functions to catalyze the synthesis of glutamine from glutamate and ammonia in an ATP-dependent reaction [23]. Genetic studies have revealed a GLUL rs10911021 polymorphism that is associated with cardiovascular disease morbidity and mortality among people with type 2 diabetes [24]. Genomewide association analyses suggested that the GLUL may regulate the risk of coronary heart disease by affecting glutamate/glutamine metabolism and the activity of the $\gamma$-glutamine cycle [25]. Coronary heart disease is the primary cause of death in patients with diabetes, and genetic factors can also act as risk factors for increased mortality. Clinical studies from European populations indicate that SNP rs10911021 is an independent risk factor for all-cause mortality in patients with 


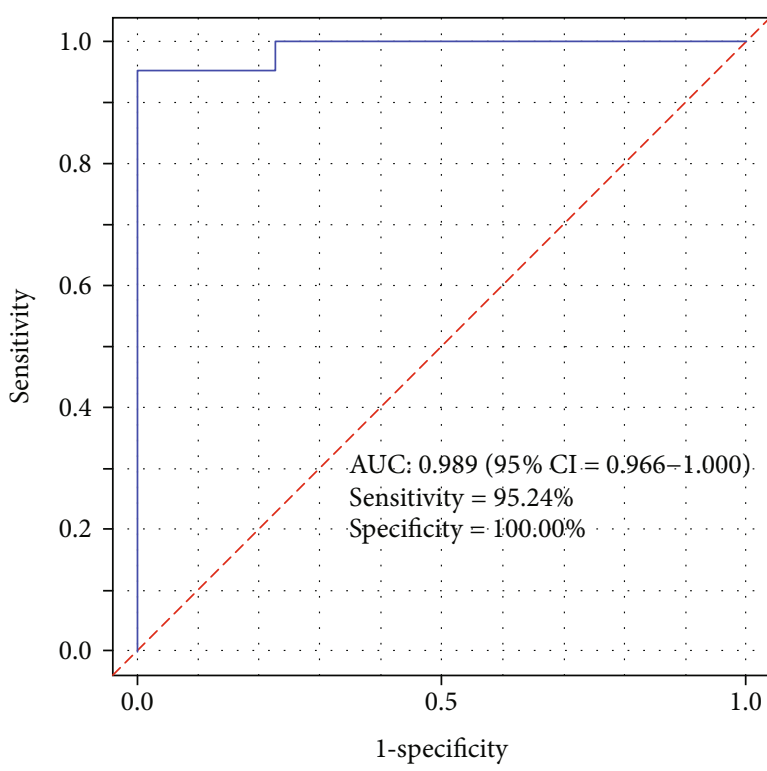

(a)

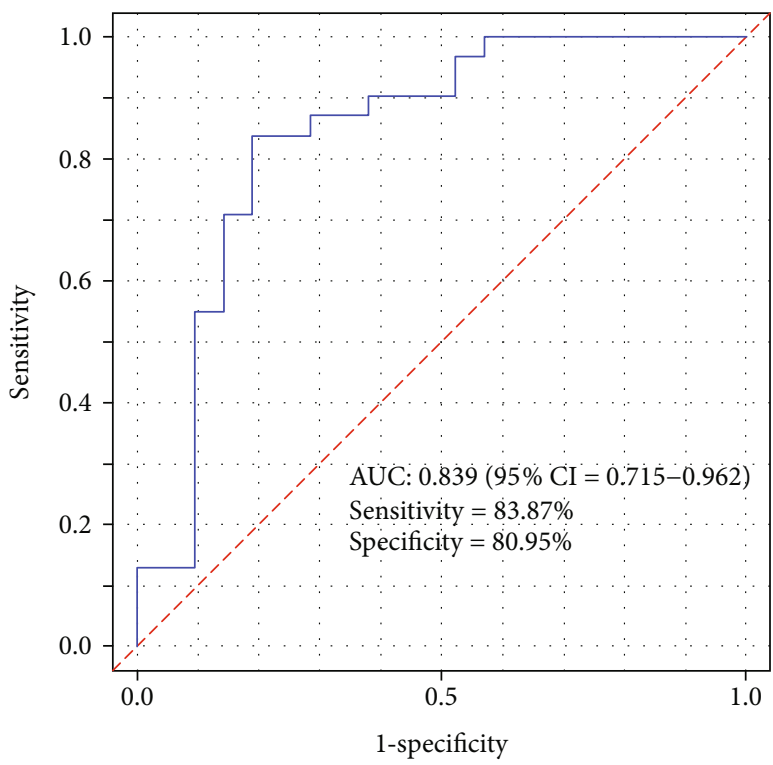

(c)

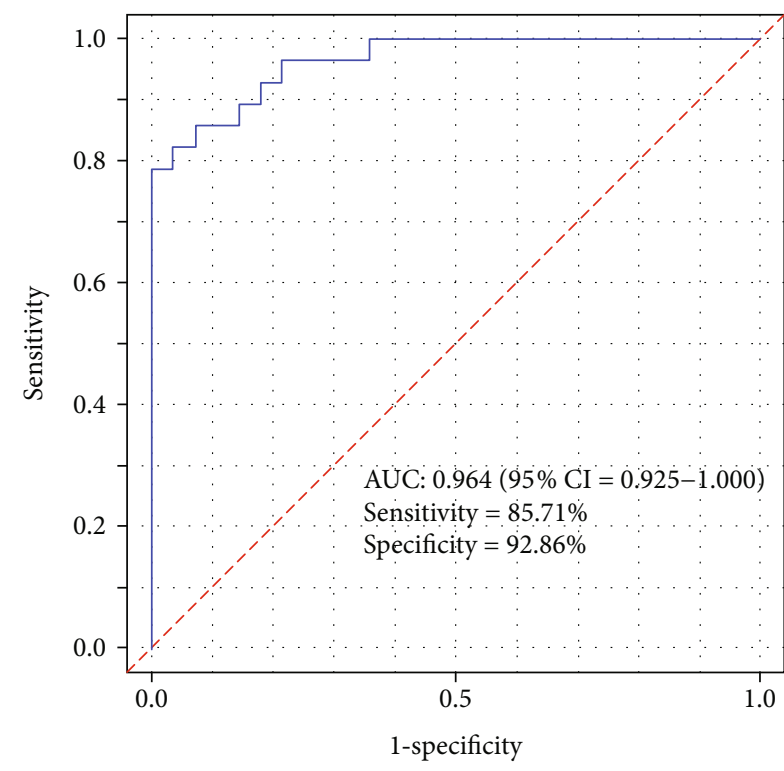

(b)

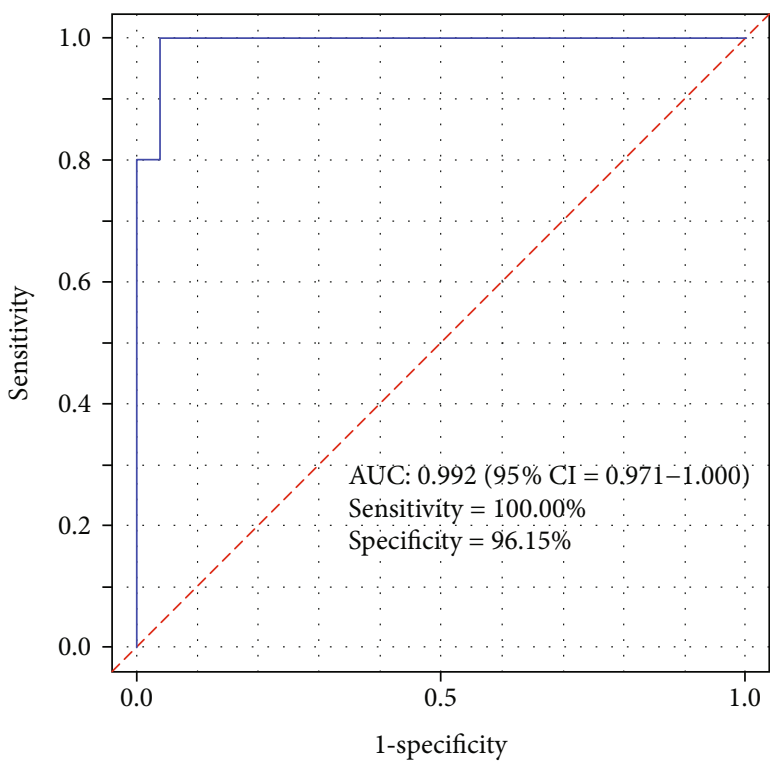

(d)

FIGURE 5: Receiver operating characteristic curves of support vector machine classifier for the discovery cohort (a), the validation cohort (b), the independent external cohort (c), and ROC analysis of the SVM classifier for AMI recurrent prediction in the independent external cohort (d).

type 2 diabetes, and the risk may be due to cardiovascular disease [26]. GLUL has also been reported to be involved in endothelial cell motility, a process that affects endothelial cell junctional integrity [27]. These studies provide possible explanations for the role of GULU in AMI.

The phosphodiesterase $4 \mathrm{~B}(P D E 4 B)$ gene is a member of the type IV cAMP-specific, cyclic nucleotide phosphodiesterase (PDE) family that regulates the cellular concentrations of cyclic nucleotides and thereby plays a role in signal transduction. In the myocardium, the PDE3 and PDE4 families are primarily used to degrade cAMP and regulate excitationcontraction coupling (ECC). PDE4 is responsible for $40 \%$ of cAMP-hydrolysis activity in human heart tissue [28]. Animal experiments have demonstrated that the primary function of PDE4 is the control of the cAMP signal, and PDE4 is responsible for the majority of the cAMP degradation activity in rat ventricular cells $[29,30]$. In the heart, cAMP regulates contraction, relaxation, and autonomy. When the regulation of this molecule is imbalanced, it significantly promotes the development of heart disease. Wang et al. demonstrated that a moderate increase in cAMP levels prevents the $\mathrm{Ca} 2+$-induced mitochondrial permeability transition pore (MPTP) from opening through Epac1, thus affecting the death of cardiomyocytes [31]. A number of studies have also confirmed that PDE4 is related to arrhythmia and heart failure [32-34]. These studies may reveal the molecular mechanism of $P D E 4 B$ in AMI; however, more detailed mechanisms require further exploration. 


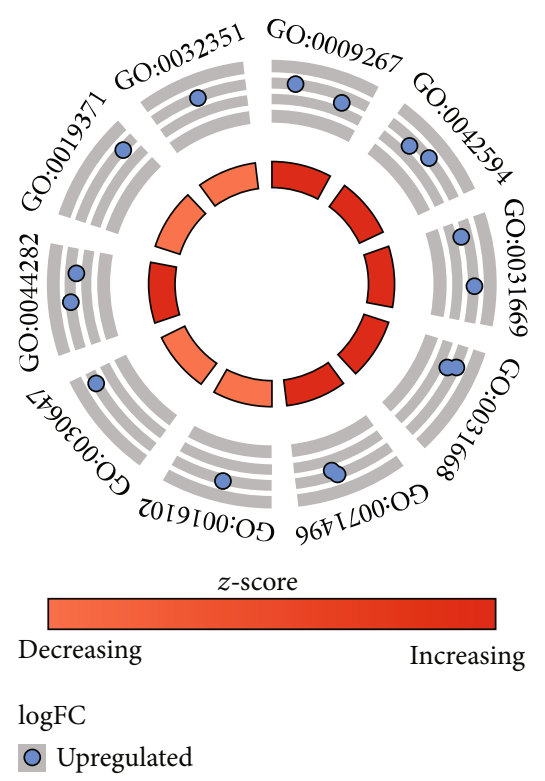

$\log \mathrm{FC}$

\begin{tabular}{|c|c|}
\hline ID & Description \\
\hline GO:0009267 & Cellular response to starvation \\
\hline GO:0042594 & Response to starvation \\
\hline GO:0031669 & Cellular response to nutrient levels \\
\hline GO:0031668 & Cellular response to extracellular stimulus \\
\hline GO:0071496 & Cellular response to external stimulus \\
\hline GO:0016102 & Diterpenoid biosynthetic process \\
\hline GO:0030647 & Aminoglycoside antibiotic metabolic process \\
\hline GO:0044282 & Small molecule catabolic process \\
\hline GO:0019371 & Cyclooxygenase pathway \\
\hline GO:0032351 & Negative regulation of hormone metabolic process \\
\hline
\end{tabular}

(a)

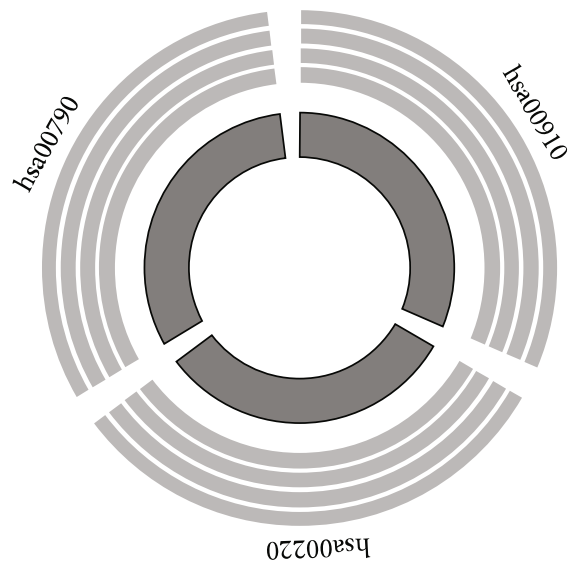

\begin{tabular}{|c|c|}
\hline ID & Description \\
\hline hsa00910 & Nitrogen metabolism \\
\hline hsa00220 & Arginine biosynthesis \\
\hline hsa00790 & Folate biosynthesis \\
\hline
\end{tabular}

(b)

Figure 6: GO and KEGG pathway analysis of identified feature genes.

The aldo-keto reductase family 1 member C3 (AKR1C3) gene is the only $17 \beta-H S D$ that is not a short-chain dehydrogenase/reductase, and this gene encodes a member of the aldose/ketoreductase superfamily. AKR1C3 has been confirmed to play a regulatory role in a variety of endocrine diseases [35]. $\mathrm{Su}$ et al. confirmed that $A K R 1 C 3$ modulates vasodilation and vasoconstriction by regulating the biosynthesis of prostaglandins [36]. Prostacyclin is a vasodilator that can inhibit platelet activation. Imbalances in prostacyclin production can result in an increased risk for coronary events [37]. AKR1C3 did not show a significant difference in expression in AMI and control in the validation dataset GSE48060. Based on this, AKR1C3 may influence AMI; however, given the small number of research studies examining this process, more research is required to provide a fuller understanding of the relationship between AKR1C3 and AMI.

As revealed in the GO and KEGG pathway analyses, these featured genes were primarily enriched in metabolismrelated processes, thus indicating that metabolism-related genes play an important role in AMI. As an important process of lipid metabolism, autophagy is activated by starvation $[38,39]$. Autophagy is also a regulated pathway of cellular deprivation [40]. Prostaglandins have been reported to be related to AMI [41, 42]. Thyroid hormone exerts an important therapeutic effect by reducing the infarct size and improving myocardial function after acute myocardial infarction [43]. Clinical studies have shown that in adults with hypertension, both low folate and high folate levels are associated with an increased risk for death from cardiovascular disease [44]. Arginine has also been confirmed to be associated with AMI $[45,46]$.

Our study does possess certain limitations. First, we failed to validate the discriminatory ability of the SVM classifier in the independent cohort with respect to the recurrent event. Second, although the ROC analysis of the support vector machine classifier for AMI recurrence prediction yields good results, the sample size for recurrent AMI was small, and its accuracy requires further verification using larger sample 
sizes. Finally, it should be noted that this research was based on bioinformatics analyses. Therefore, further validations in vivo and in vitro are required.

\section{Conclusion}

The present study identified three metabolic-related genes (GLUL, PDE4B, and AKR1C3) in patients with AMI, and these genes may be useful as potential biomarkers in the diagnosis of AMI. Knowledge of these genes will improve our understanding of the molecular mechanism underlying the occurrence of AMI.

\section{Data Availability}

The raw data of this study are derived from the GEO data portal (https://www.ncbi.nlm.nih.gov/geo/; accession numbers: GSE66360 and GSE68060), which is a publicly available database.

\section{Conflicts of Interest}

The authors declare that they have no conflicts of interest.

\section{Authors' Contributions}

Hang Xie and Enfa Zhao contributed equally to this work and should be considered co-first authors.

\section{Supplementary Materials}

Supplementary table 1: reference gene set. (Supplementary Materials)

\section{References}

[1] J. Guo, H.-B. Liu, C. Sun et al., "MicroRNA-155 promotes myocardial infarction-induced apoptosis by targeting RNAbinding protein QKI," Oxidative Medicine and Cellular Longevity, vol. 2019, Article ID 4579806, 14 pages, 2019.

[2] M. Cohen, G. F. Gensini, F. Maritz et al., "Prospective evaluation of clinical outcomes after acute ST-elevation myocardial infarction in patients who are ineligible for reperfusion therapy: preliminary results from the TETAMI registry and randomized trial," Circulation, vol. 108, no. 90161, pp. 14III--14II21, 2003.

[3] E. Braunwald, "Unstable angina and non-ST elevation myocardial infarction," American Journal of Respiratory and Critical Care Medicine, vol. 185, no. 9, pp. 924-932, 2012.

[4] J. P. Park, M. K. Park, and J. W. Yun, "Proteomic biomarkers for diagnosis in acute myocardial infarction," Biomarkers, vol. 16, no. 1, pp. 1-11, 2010.

[5] J. Kim, N. Ghasemzadeh, D. J. Eapen et al., "Gene expression profiles associated with acute myocardial infarction and risk of cardiovascular death," Genome Medicine, vol. 6, no. 5, p. $40,2014$.

[6] D. M. Lloyd-Jones, "Cardiovascular risk prediction: basic concepts, current status, and future directions," Circulation, vol. 121, no. 15, pp. 1768-1777, 2010.

[7] G. D. Lewis, A. Asnani, and R. E. Gerszten, "Application of metabolomics to cardiovascular biomarker and pathway dis- covery," Journal of the American College of Cardiology, vol. 52, no. 2, pp. 117-123, 2008.

[8] E. M. Rogg, W. T. Abplanalp, C. Bischof et al., "Analysis of cell type-specific effects of microRNA-92a provides novel insights into target regulation and mechanism of action," Circulation, vol. 138, no. 22, pp. 2545-2558, 2018.

[9] X. Lin, F. Yang, L. Zhou et al., "A support vector machinerecursive feature elimination feature selection method based on artificial contrast variables and mutual information," Journal of Chromatography. B, Analytical Technologies in the Biomedical and Life Sciences, vol. 910, pp. 149-155, 2012.

[10] V. Cherkassky, "The nature of statistical learning theory ," IEEE Transactions on Neural Networks, vol. 8, no. 6, p. 1564, 1997.

[11] I. Guyon, "Erratum: gene selection for cancer classification using support vector machines," Machine Learning, vol. 46, no. 1-3, pp. 389-422, 2001.

[12] M. Pirooznia, J. Y. Yang, M. Q. Yang, and Y. Deng, “A comparative study of different machine learning methods on microarray gene expression data," BMC Genomics, vol. 9, Supplement 1, p. S13, 2008.

[13] S. Mahadevan, S. L. Shah, T. J. Marrie, and C. M. Slupsky, "Analysis of metabolomic data using support vector machines," Analytical Chemistry, vol. 80, no. 19, pp. 75627570, 2008.

[14] G. Yu, L. G. Wang, Y. Han, and Q. Y. He, “clusterProfiler: an R package for comparing biological themes among gene clusters," OMICS, vol. 16, no. 5, pp. 284-287, 2012.

[15] S. Boateng and T. Sanborn, "Acute myocardial infarction," Disease-a-Month, vol. 59, no. 3, pp. 83-96, 2013.

[16] H. Jiang, D. Jia, B. Zhang et al., "Exercise improves cardiac function and glucose metabolism in mice with experimental myocardial infarction through inhibiting HDAC4 and upregulating GLUT1 expression," Basic Research in Cardiology, vol. 115, no. 3, p. 28, 2020.

[17] J. B. N. Moreira, M. Wohlwend, S. Fenk et al., "Exercise reveals proline dehydrogenase as a potential target in heart failure," Progress in Cardiovascular Diseases, vol. 62, no. 2, pp. 193202, 2019.

[18] C. J. Willer, S. Sanna, A. U. Jackson et al., "Newly identified loci that influence lipid concentrations and risk of coronary artery disease," Nature Genetics, vol. 40, no. 2, pp. 161-169, 2008.

[19] R. Iqbal, N. Jahan, Y. Sun, and H. Xue, "Genetic association of lipid metabolism related SNPs with myocardial infarction in the Pakistani population," Molecular Biology Reports, vol. 41, no. 3, pp. 1545-1552, 2014.

[20] C. B. Anea, M. Zhang, D. W. Stepp et al., "Vascular disease in mice with a dysfunctional circadian clock," Circulation, vol. 119, no. 11, pp. 1510-1517, 2009.

[21] K. F. Storch, O. Lipan, I. Leykin et al., "Extensive and divergent circadian gene expression in liver and heart," Nature, vol. 417, no. 6884, pp. 78-83, 2002.

[22] Z. Zhu, B. Hua, Z. Shang et al., "Altered clock and lipid metabolism-related genes in atherosclerotic mice kept with abnormal lighting condition," BioMed Research International, vol. 2016, Article ID 5438589, 14 pages, 2016.

[23] Y. Wang, S. Fan, J. Lu et al., "GLUL promotes cell proliferation in breast cancer," Journal of Cellular Biochemistry, vol. 118, no. 8, pp. 2018-2025, 2017. 
[24] H. Tang and C. Gao, "Comment on The Look AHEAD Research Group. Prospective association ofGLULrs10911021 with cardiovascular morbidity and mortality among individuals with type 2 diabetes: the Look AHEAD Study. Diabetes 2016;65:297-302," Diabetes, vol. 65, no. 9, p. e29, 2016.

[25] L. Qi, Q. Qi, S. Prudente et al., “Association between a genetic variant related to glutamic acid metabolism and coronary heart disease in individuals with type 2 diabetes," JAMA, vol. 310, no. 8, pp. 821-828, 2013.

[26] S. Prudente, H. Shah, D. Bailetti et al., "Genetic variant at the GLUL locus predicts all-cause mortality in patients with type 2 diabetes," Diabetes, vol. 64, no. 7, pp. 2658-2663, 2015.

[27] G. Eelen, C. Dubois, A. R. Cantelmo et al., "Role of glutamine synthetase in angiogenesis beyond glutamine synthesis," Nature, vol. 561, no. 7721, pp. 63-69, 2018.

[28] D. Mika, P. Bobin, M. Lindner et al., "Synergic PDE3 and PDE4 control intracellular cAMP and cardiac excitationcontraction coupling in a porcine model," Journal of Molecular and Cellular Cardiology, vol. 133, pp. 57-66, 2019.

[29] J. Leroy, A. Abi-Gerges, V. O. Nikolaev et al., "Spatiotemporal dynamics of $\beta$-Adrenergic cAMP signals and L-type $\mathrm{Ca} 2$ +Channel regulation in adult rat ventricular Myocytes," Circulation Research, vol. 102, no. 9, pp. 1091-1100, 2008.

[30] M. Mongillo, T. McSorley, S. Evellin et al., "Fluorescence resonance energy transfer-based analysis of cAMP dynamics in live neonatal rat cardiac myocytes reveals distinct functions of compartmentalized phosphodiesterases," Circulation Research, vol. 95, no. 1, pp. 67-75, 2004.

[31] Z. Wang, D. Liu, A. Varin et al., "A cardiac mitochondrial cAMP signaling pathway regulates calcium accumulation, permeability transition and cell death," Cell Death \& Disease, vol. 7, no. 4, p. e2198, 2016.

[32] C. E. Molina, J. Leroy, W. Richter et al., "Cyclic adenosine monophosphate phosphodiesterase type 4 protects against atrial arrhythmias," Journal of the American College of Cardiology, vol. 59, no. 24, pp. 2182-2190, 2012.

[33] P. Bobin, A. Varin, F. Lefebvre, R. Fischmeister, G. Vandecasteele, and J. Leroy, "Calmodulin kinase II inhibition limits the pro-arrhythmic Ca2+ waves induced by cAMPphosphodiesterase inhibitors," Cardiovascular Research, vol. 110, no. 1, pp. 151-161, 2016.

[34] S. Karam, J. P. Margaria, A. Bourcier et al., "Cardiac overexpression of $\mathrm{PDE} 4 \mathrm{~B}$ blunts $\beta$-adrenergic response and maladaptive remodeling in heart failure," Circulation, vol. 142, no. 2, pp. 161-174, 2020.

[35] T. M. Penning, "AKR1C3 (type 5 17 $\beta$-hydroxysteroid dehydrogenase/prostaglandin $\mathrm{F}$ synthase): roles in malignancy and endocrine disorders," Molecular and Cellular Endocrinology, vol. 489, pp. 82-91, 2019.

[36] E. J. Su, L. Ernst, N. Abdallah et al., "Estrogen receptor- $\beta$ and fetoplacental endothelial prostanoid biosynthesis: a link to clinically demonstrated fetal growth restriction," The Journal of Clinical Endocrinology and Metabolism, vol. 96, no. 10, pp. E1558-E1567, 2011.

[37] P. M. Kearney, C. Baigent, J. Godwin, H. Halls, J. R. Emberson, and C. Patrono, "Do selective cyclo-oxygenase-2 inhibitors and traditional non-steroidal anti-inflammatory drugs increase the risk of atherothrombosis? Meta-analysis of randomised trials," BMJ, vol. 332, no. 7553, pp. 1302-1308, 2006.

[38] R. Singh, S. Kaushik, Y. Wang et al., "Autophagy regulates lipid metabolism," Nature, vol. 458, no. 7242, pp. 1131-1135, 2009.
[39] N. Mizushima, “Autophagy: process and function," Genes \& Development, vol. 21, no. 22, pp. 2861-2873, 2007.

[40] D. J. Klionsky and S. D. Emr, "Autophagy as a regulated pathway of cellular degradation," Science, vol. 290, no. 5497, pp. 1717-1721, 2000.

[41] C. W. Sung, J. H. Jung, S. H. Lee et al., "Acute myocardial infarction due to vasospasm induced by prostaglandin," The Canadian Journal of Cardiology, vol. 25, no. 10, pp. e359e360, 2009.

[42] A. A. Elesber, P. J. Best, R. J. Lennon et al., "Plasma 8-isoprostaglandin F2alpha, a marker of oxidative stress, is increased in patients with acute myocardial infarction," Free Radical Research, vol. 40, no. 4, pp. 385-391, 2009.

[43] A. Jabbar, A. Pingitore, S. H. S. Pearce, A. Zaman, G. Iervasi, and S. Razvi, "Thyroid hormones and cardiovascular disease," Nature Reviews. Cardiology, vol. 14, no. 1, pp. 39-55, 2017.

[44] S. Nkemjika, E. Ifebi, L. T. Cowan et al., "Association between serum folate and cardiovascular deaths among adults with hypertension," European Journal of Clinical Nutrition, vol. 74, no. 6, pp. 970-978, 2020.

[45] P. Tripathi, M. K. Misra, and S. Pandey, "Role of l-arginine on dyslipidemic conditions of acute myocardial infarction patients," Indian Journal of Clinical Biochemistry, vol. 27, no. 3, pp. 296-299, 2012.

[46] M. Du, W. Yang, S. Schmull, J. Gu, and S. Xue, "Inhibition of peptidyl arginine deiminase- 4 protects against myocardial infarction induced cardiac dysfunction," International Immunopharmacology, vol. 78, p. 106055, 2020. 